\title{
50 años de cirugía de bypass coronario
}

Señor Editor:

He leído con gran interés el artículo "50 AÑOS DE CIRUGÍA DE BYPASS CORONARIO” publicado en el último número de la Revista Chilena de Cardiología. En el mismo, el Dr. Ricardo Zalaquett hace un breve, pero muy interesante recorrido por los que, en su consideración, son los primeros 50 años de la cirugía de puentes aorto-coronarios. ${ }^{1}$

Sentenciar que en el 2017 la cirugía de bypass coronario cumplió medio siglo de existencia puede ser motivo de debate. Así, en el excelente artículo del Dr. Zalaquett hay dos nombres que no deben ser omitidos de la historia de la cirugía de revascularización miocárdica que quizás obligan a replantear la fecha que debiera tomarse para festejar el cumpleaños de este procedimiento el de los doctores William Polk Longmire Jr. y Robert Hans Goetz.
Longmire es indudablemente uno de los padres de la cirugía cardíaca-aunque fue sobre todo un destacadísimo cirujano general-y uno de los hombres de mente más abierta y propensa a la innovación dentro de la práctica quirúrgica en general. Desde fecha muy temprana, el 29 de noviembre de 1944, entró en la historia de la cardiocirugía cuando, junto a Denton Cooley, asistió a Alfred Blalock en la la primera anastomosis entre las arterias subclavia y pulmonar ${ }^{2}$. En 1958 reporta, en primicia, una técnica para la endarterectomía coronaria bajo visión directa y es presuntamente también por ese tiempo que realiza la primera anastomosis entre la arteria mamaria interna y un vaso coronario en humanos, aunque este hecho se mantuvo desconocido por más de tres décadas. En una comunicación personal de Longmire en 1990, describe cómo a comienzos del 58 , al menos un par de veces se vio obligado a efectuar un puente entre un segmento distal de la coronaria de- 
recha y la mamaria interna. Durante la realización de trombo-endarterectomías el vaso coronario, al parecer severamente calcificado, prácticamente se le desintegró en las manos, y decidió, junto a su ayudante Jack Cannon, anastomosar los vasos mencionados en un intento por salvar la vida de su paciente. ${ }^{3}$ Curiosamente, como casi todos los grandes inventos del hombre, la cirugía de revascularización coronaria había nacido de la necesidad, la casualidad y los contratiempos.

Goetz llegó posteriormente a especializarse en Sudáfrica en temas relacionados con fisiología y cirugía torácica y vascular ${ }^{5,6}$ y finalmente se radicó en Estados Unidos en el año 1957. Para 1960 había retomado las ideas de su compatriota Payr, de principios de siglo, ${ }^{7}$ $\mathrm{y}$ al año siguiente publicaba la primera serie en perros, de anastomosis sin suturas entre la mamaria interna izquierda y las coronarias descendente anterior y circunfleja. ${ }^{8}$ Sin embargo, mientras su artículo esperaba ser publicado, el 2 de mayo de 1960, impulsado por sus fabulosos resultados experimentales, en el Hospital Van Etten, del Bronx, a un taxista de 38 años se le realizó la primera cirugía planificada de bypass coronario. Mediante una esternotomía media, con una técnica sin suturas, empleando un anillo de tántalo, Goetz, auxiliado por Rohman y Haller, en tan solo 17 segundos anastomosó las arterias mamaria interna y coronaria derecha de su paciente. ${ }^{4}$

Curiosamente, la operación que presuntamente debió provocar un cambio de era en la cirugía cardíaca se convirtió en el único procedimiento de este tipo ejecutado por estos galenos. A pesar de que a los 14 días de la intervención se demostró la permeabilidad del injerto y que el paciente permaneció asintomático durante más de un año, al parecer la comunidad científica mundial, pero en especial la que rodeaba más cercanamente a
Goetz, no estaba preparada para un salto tan enorme, y se opuso violentamente al procedimiento, principalmente por el hecho de que el paciente falleciera de un infarto miocárdico de cara posterior, casi a los 14 meses de la operación. ${ }^{4}$ Tendrían que pasar 4 años para que en la antigua Unión Soviética, Vasilii Ivanovich Kolesovvolviera a intentar el procedimiento, y un poco más para que George Green fuera aclamado como el supuesto iniciador de la técnica en Estados Unidos. ${ }^{9}, 10$

Este año, algunos autores han celebrado los 50 años del nacimiento de la cirugía de bypass coronario, asumiendo como cimientos de la misma la práctica de Favaloro en Cleveland en 1967.11 Este magnífico galeno argentino es indudablemente otro de los padres de la cirugía cardíaca moderna, y sus injertos de vena safena colocaron en el primer plano de la práctica quirúrgica a la cirugía coronaria, y le pavimentaron su futuro camino. Sin embargo, aseverar que el nacimiento de la cirugía de puentes coronarios nació con Favaloro, no rendiría adecuado tributo a la verdad histórica.

La tercera acepción de la palabra "nacer", según el Diccionario de la Real Academia Española la define como "empezar a salir de su semilla". La simiente del bypass coronario, germinó un poco antes de que Favaloro decidiera continuar regándola. Los menos suspicaces situarán el nacimiento en las manos de Longmire, en Los Ángeles, quizás en una fría tarde del invierno de 1958; otros la verán brotar en la ciudad que nunca duerme, en el mes en que retoñan las flores al norte del ecuador, quizás en una cálida mañana de 1960.

Dr. Yoandy López de la $C$.

Hospital Universitario Cardiocentro Ernesto Guevara Santa Clara. Villa Clara. República de Cuba. 
1. ZALAQUETT R. 50 AÑOS DE CIRUGíA DE BYPASS CORONARIO. Meditar el pasado, enfrentar el presente y forjar el futuro. Rev Chil Cardiol 2017;36:162-9.

2. LOWENSTEIN E, REVES JG. A History of Cardiac Anesthesiology. In: Eger II EI, al e, editors. The Wondrous Story of Anesthesia. New York: Springer; 2014. p. 829-46.

3. PICICHÉ M. The History of Myocardial Revascularization Before the Advent of Cardiopulmonary Bypass. In: Piciché M, editor. Dawn and Evolution of Cardiac Procedures: Research Avenues in Cardiac Surgery and Interventional Cardiology. Milan: Springer-Verlag; 2013. p. 65-77.

4. STEPHENSON LW. History of Cardiac Surgery. In: Cohn L, editor. Cardiac Surgery in the Adult. New York: McGraw-Hill; 2008. p. 3-28.

5. KONSTANTINOV IE. ROBERT H. GOETZ: The Surgeon Who Performed the First Successful Clinical Coronary Artery Bypass Operation. Ann Thorac Surg. 2000;69:1966-72.

6. GOETZ RH, MARR JAS. The importance of the second thoracic ganglion for the sympathetic supply of the upper extremities-two new approaches for its removal. Clin Proc 1944;3:102-14.

7. BISCHOF G, PANHOFER P, NEUMAYER C. Endoscopic Sympathetic Surgery. In: Inderbitzi RGC, Schmid RA, Melfi FMA, Casula RP, editors. Minimally Invasive Thoracic and Cardiac Surgery. Berlin: Springer; 2012. p. 275-99.

8. TOZZI P. Historical overview of vascular anastomoses. Sutureless anastomoses: Secrets for Success. Darmstadt: Springer; 2007. p. 1-11.

9. SAJJA L R, MANNAM G. Internal thoracic artery: Anatomical and biological characteristics revisited. Asian Cardiovascular \& Thoracic Annals. 2015;23(1):88-99.

10. AL-ATASSI T, TOEG HD, CHAN V, RUEL M. Coronary Artery Bypass Grafting In: Sellke FW, editor. Sabiston \& Spencer surgery of the chest. 9th ed. Philadelphia: Elsevier; 2016. p. 1551-88.

11. FAVALORO RG. Surgical treatment of coronary arteriosclerosis. The Williams \& Wilkins Company. Baltimore, 1970 